Bradley, S. G. (1958). J. gen. Microbiol. 18, 591-596

\title{
Mechanism of 'Vegetative Hybridization' in Streptomyces
}

\author{
BY S. G. BRADLEY \\ Department of Bacteriology and Immunology, University of Minnesota, \\ Minneapolis 14, Minnesota, U.S.A.
}

SUMMARY: A population of Streptomyces griseus, strain W 107, exposed for several growth cycles to a sterile culture filtrate of $S$. griseus, strain M142, acquired several genetic characteristics similar to those of strain M142. The changes observed were: (1) streptomycin sensitivity to resistance; (2) bacteriophage $V_{1}$ sensitivity to resistance; (3) absence to presence of soluble pigment; (4) presence to absence of pigment in the vegetative mycelium. The filtrate contained streptomycin and a temperate bacteriophage. The low concentration of streptomycin did not grossly inhibit the growth of strain W 107 but streptomycin-resistant mutants were selected. Resistance to the temperate phage frequently conferred resistance to bacteriophage $V_{1}$. The observed morphological changes were coupled with bacteriophage- and streptomycinsusceptibility. Vegetative hybridization was the result of selection of mutants rather than gene transfer.

The streptomycetes are filamentous bacteria which resemble the true moulds in many respects. Hyphal fusion with resulting heterokaryosis has been demonstrated for several true moulds (Beadle \& Coonradt, 1944) and for different species of Streptomyces (Bradley, 1957a). Specialized sexual structures have not been found in the streptomycetes. Sexuality in Streptomyces (Sermonti \& Spada-Sermonti, 1956) probably resembles either parasexuality in Aspergillus or sexuality in Escherichia coli (Lederberg, 1952).

As is the case among true bacteria, many streptomycetes are lysogenic (Bradley, 1957b; Welsch, 1956). Temperate streptomyces-phages might serve as vectors for the transfer of genetic material. Alteration of the genetic composition of Streptomyces populations by extracts which might contain bacteriophage has been reported (Horvath, Marton \& Oroszlan, 1954). Several growth cycles in the presence of the extract were required before phenotypic shifts were detected. The characteristics affected were colonial morphology, pigmentation and antibiotic production. The mechanism responsible for this 'vegetative hybridization' was not amply studied. Similar changes in populations of Streptomyces griseus treated with a culture filtrate have now been observed. These phenotypic shifts might result from (1) phage-mediated transduction; (2) deoxyribonucleic acid-mediated transformation; (3) selection for particular phenotypes. This paper presents evidence that the culture filtrate used contained selective agents. Some of these have now been identified.

\section{METHODS}

Mutant substrains of Steptomyces griseus were used throughout this investigation. The genetic markers employed were growth-factor requirements, antibiotic sensitivity, bacteriophage sensitivity and morphology. The 
characteristics of the mutant and ancestral substrains are shown in Table 1. The mutant substrains were obtained after ultraviolet irradiation or by direct selection using methods previously described (Bradley \& Lederberg, 1956).

Table 1. Principal stocks of Streptomyces griseus used and their ancestors

$\begin{array}{lc}\text { Strain } & \text { Derived from } \\ \text { W 86 } & \text { Nature } \\ \text { W 107 } & \text { W86 } \\ \text { W 104 } & \text { Nature } \\ \text { M 142 } & \text { W 104 }\end{array}$

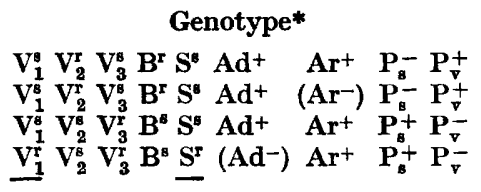

* $V^{s}$ or $V^{r}$ denotes sensitivity or resistance to bacteriophage. $V_{1}, V_{2}$ and $V_{3}$ represent bacteriophages WSP-3, WSP-2 and MSP-2 respectively. $B^{s}$ or $B^{r}$ denotes sensitivity or resistance to 2500 units bacitracin $/ 1$. $S^{8}$ or $S^{x}$ denotes sensitivity or resistance to $25 \mathrm{mg}$. dihydrostreptomycin sulphate/l. $\mathrm{Ad}^{+}$or $\mathrm{Ad}^{-}$and $\mathrm{Ar}^{+}$or $\mathrm{Ar}^{-}$denote independence or dependence for exogenous adenine or arginine $20 \mathrm{mg} / \mathrm{L} . \mathrm{P}_{\mathrm{s}}^{+}$or $\mathrm{P}_{\mathrm{s}}^{-}$and $\mathrm{P}_{v}^{+}$or $\mathrm{P}_{v}^{-}$denote presence or absence of soluble pigment and pigment in the vegetative mycelium respectively.

( ), genetic markers obtained after ultraviolet irradiation.

, genetic markers obtained by direct selection.

The basal medium was composed of: glucose, $20 \mathrm{~g} ; \mathrm{KNO}_{3}, 2 \mathrm{~g}$.; $\mathrm{K}_{2} \mathrm{HPO}_{4}$, 2 g.; $\mathrm{MgSO}_{4} .7 \mathrm{H}_{2} \mathrm{O}, 0.5$ g.; $\mathrm{CaCO}_{3}, 0 \cdot 25$ g.; water, 11 . For complete medium, $0.1 \%(\mathrm{w} / \mathrm{v})$ Difco yeast extract was added. The medium was solidified with $1.5 \%(w / v)$ agar. Glucose and salts solutions were autoclaved separately at double strength.

The culture filtrate was prepared as follows. Complete medium was inoculated with Streptomyces griseus, strain M 142, to yield $10^{6}-10^{7}$ spores $/ \mathrm{ml}$. and incubated at $30^{\circ}$ on a rotary shaker. The culture underwent spontaneous lysis after 4 days and the culture was then filtered through coarse filter-paper to remove floating debris and remaining mycelium. The culture filtrate was further clarified by centrifugation and rendered bacteriologically sterile by filtration.

\section{RESULTS}

Streptomyces griseus, strain W107, was grown in complete medium to which $5 \%(\mathrm{v} / \mathrm{v})$ strain M 142 filtrate had been added. After several serial subcultures exposed to the filtrate, non-parental types appeared, with respect to the streptomycin and bacteriophage $V_{1}$ characters. The strain M142 culture filtrate did not affect the bacteriophage $V_{3}$ and arginine characters (Table 2). These data, taken from a typical experiment, have only slight quantitative significance because 'colony forming unit' is not a distinct entity. Colony forming unit refers to a hyphal fragment of any size which produces a single discrete colony. The number of subcultures required to establish non-parental types varied considerably as between different experiments. Rarely nonparental types arose as early as the second subculture and in one experiment, non-parental types did not become established until the twelfth subculture.

Treated strain W107, selected for one non-parental character, frequently differed for other characters as well (Table 3). Growth-factor requirements, bacitracin sensitivity and bacteriophage $\mathrm{V}_{3}$ sensitivity were not altered in the 
selected streptomycin- or bacteriophage $V_{1}$-resistant substrains. Other factors were affected to different degrees, exceeding the spontaneous mutation rate, which was less than $10^{-3}$.

Table 2. Selection for non-parental phenotypes of Streptomyces griseus strain $W 107$



in strain
M142 filtrate

0
1
2
3
4
5
6



$\overbrace{S^{r}}^{\begin{array}{l}V_{1}^{r} \\ \text { Colonies } / 10^{7} \text { colony-forming units }\end{array}} A^{V_{3}^{r}}$

\begin{tabular}{rrrr}
\hline 0 & 20 & 7 & 0 \\
0 & 31 & 4 & 0 \\
0 & 18 & 4 & 0 \\
0 & 59 & 9 & 0 \\
27 & 132 & 11 & 0 \\
897 & 1021 & 3 & 0 \\
$3.3 \times 10^{6}$ & $1.7 \times 10^{6}$ & 0 & 0
\end{tabular}

Table 3. Characteristics of non-parental isolates of Streptomyces griseus strain W107

Strain W107 was grown for 9 subcultures in the presence of $5 \%(v / v)$ strain $M 142$ or W 104 culture-filtrates.

\begin{tabular}{|c|c|c|c|c|c|c|c|c|c|c|}
\hline \multirow{3}{*}{$\begin{array}{l}\text { Filtrate } \\
\text { used }\end{array}$} & \multirow{3}{*}{$\begin{array}{l}\text { Character } \\
\text { selected }\end{array}$} & \multicolumn{9}{|c|}{ Character } \\
\hline & & $\mathbf{V}_{1}$ & $\mathbf{v}_{\mathbf{2}}$ & $\mathbf{V}_{\mathbf{3}}$ & B & $\mathbf{S}$ & $\mathbf{P}_{\mathbf{s}}$ & $\mathbf{P}_{\mathbf{v}}$ & Ad & $\overrightarrow{A r}$ \\
\hline & & \multicolumn{9}{|c|}{$\%$ different from strain $\mathrm{W} 107$} \\
\hline M 142 & $\begin{array}{c}\text { Control, no } \\
\text { selection }\end{array}$ & 13 & 4 & $<0 \cdot 1$ & $<0 \cdot 1$ & 35 & 2 & 9 & $<0 \cdot 1$ & $<0 \cdot 1$ \\
\hline M 142 & $\mathbf{S}^{\mathbf{x}}$ & 27 & $<0 \cdot 1$ & $<0.1$ & $<0.1$ & 100 & 9 & 14 & $<0 \cdot 1$ & $<0.1$ \\
\hline M 142 & $V_{1}^{r}$ & 100 & 38 & $<0.1$ & $<0 \cdot 1$ & 13 & $0 \cdot 6$ & 15 & $<0.1$ & $<0 \cdot 1$ \\
\hline W 104 & $\begin{array}{l}\text { Control, no } \\
\text { selection }\end{array}$ & $\mathbf{2 4}$ & 7 & 0.5 & $<0 \cdot 1$ & $<0 \cdot 1$ & $<0 \cdot 1$ & $\mathbf{2}$ & $<0 \cdot 1$ & $<\cdot 01$ \\
\hline
\end{tabular}

A filtrate of strain $\mathrm{M} 142$, heated at $65^{\circ}$ for $30 \mathrm{~min}$., was added to strain W 107 cultures. No strain $W_{107}$ organism, resistant to bacteriophage $V_{1}$, was found during any of the serial subcultures. The development of streptomycin resistance was not affected. This indicated that at least two distinct agents were involved.

Strain M 142 is lysogenic (Bradley, 1957c). Antisera against the temperate phage L3 was added to the strain W107 cultures, in addition to the M142 culture-filtrate. No bacteriophage $\mathrm{V}_{\mathbf{1}}$-resistant organisms were isolated during the serial subcultures. As with heat-treated filtrates, antisera did not affect the development of streptomycin resistance. The temperate phage L3 was responsible for the increased proportion of $V_{1}^{r}$ cells.

The ancestor of strain M 142, i.e. W 104, is streptomycin sensitive, phage $V_{1}$ sensitive and lysogenic for the temperate phage L3. Filtrates of strain $W 104$ added to growing cultures of $W_{107}$ produced the change from phage $V_{1}$ sensitivity to resistance as effectively as filtrates of strain M142. However, 
the W104 filtrate did not affect the streptomycin character (Table 3). As before, the number of subcultures required to attain corresponding yields of the non-parental types varied considerably as between different experiments.

Table 4. Factors affecting the activity of Streptomyces griseus strain M142 filtrates

Composition of $\mathrm{W} 107$ population after 8 subcultures



Treatment

$65^{\circ}$ for $30 \mathrm{~min}$.

Anti-L. 3 serum

Activated charcoal

pH 2 eluate from charcoal

Control, no treatment

Bacteriophages $\mathrm{L} 3$ and $\mathrm{V}_{1}$ are demonstrably different. However, resistance to L3 might concurrently confer resistance to $V_{1}$. This was shown to occur. Of 63 mutants of strain W107 selected for resistance to L3, five were also resistant to $V_{1}$. Moreover, two were resistant to $V_{3}$ and one lacked pigment in the vegetative mycelium. Resistance to the temperate phage L3 might result by selection of a mutant or by lysogenization. If W 107 were lysogenized, free phage should be demonstrable in culture autolysates. Bacteriologically sterile filtrates of phage $V_{1}$-resistant derivatives of strain $W_{107}$ were plated with sensitive indicator strains. No evidence of lysogenization was found. The temperate phage L3 was' irregularly found in lysates of strain M142. Therefore the temperate phage L3 was the selective agent responsible for certain changes in morphology and bacteriophage susceptibility.

Activated charcoal removed the factor in the strain $\mathrm{MI42}$ filtrate ( $\mathrm{pH} \mathrm{7 \cdot 0}$ ) responsible for the change from streptomycin sensitivity to resistance. An eluate made by extracting the activated charcoal with acidified water $(\mathrm{pH} 2)$ was able to alter streptomycin susceptibility. The eluate inhibited completely the growth of strain W107 unless the eluate was adequately diluted. This indicated that strain M142 might produce an antibiotic. The filtrate inhibited streptomycin-sensitive but not streptomycin-resistant strains of Escherichia coli. Comparing the inhibitory effect of the culture filtrate and dihydrostreptomycin sulphate standards, it was found that strain M142 produced antibiotic equivalent to $\mathbf{3 0 - 4 0} \mathrm{mg}$. streptomycin/1.

The low concentration of streptomycin added to the experimental flasks was not sufficient to alter grossly the growth of strain W 107. However, W107 grown for 10 subcultures in $2 \mathrm{mg}$. dihydrostreptomycin sulphate/l. became resistant to $25 \mathrm{mg}$./1. A few of these colonies on complete agar produced soluble pigment ( 7 of 1291) and several lacked pigment in the vegetative mycelium (122 of 1291).

\section{DISCUSSION}

Culture filtrates of Streptomyces griseus, strain M142, brought about certain genetic changes in $S$. griseus, strain W 107. Several growth cycles in the filtrate were required before these changes became detectable. Selective agents pro- 
duced by strain M142 were responsible. Two of the agents, temperate bacteriophage L3 and streptomycin, were isolated from the M 142 filtrates.

To demonstrate conclusively that the genesis of resistance to bacteriophage $V_{1}$ was the result of selection of mutants resistant to temperate bacteriophage L3, it was necessary to rule out DNA-mediated transformation, phage-mediated transduction and cross-resistance conferred by lysogenization. Antiserum against temperate phage $L 3$ prevented the development of $V_{1}$ resistance. Temperate phage L3 was essential, therefore DNA-mediated transformation was not the mechanism involved. Moreover, L3 grown on a $V_{1}$-sensitive donor promoted the development of $V_{1}$ resistance as effectively as phage L3 grown on strain M 142. This was inconsistent with both DNA-mediated transformation and phage-mediated transduction. Temperate phage L3 could not be isolated from autolysates of the $V_{1}$-resistant derivatives of strain $W_{107}$. This indicated that lysogenization had not occurred.

Quantitative comparisons among the streptomycetes were difficult because 'cell number' could not be accurately enumerated. Moreover, geneticallyaltered nuclei might be masked by normal nuclei in the same cytoplasm. The alterations resulting from dihydrostreptomycin sulphate and phage L 3 did not precisely duplicate the effect of the culture filtrate. It is possible that other selective agents were involved. Metabolic products such as alanine and kinetin select for particular phenotypes of Brucella (Mika, Braun, Ciaccio \& Goodlow, 1954; Braun, Firshein \& Whallon, 1957). Horvath et al. (1954) interpreted similar data as transduction or transformation. Selection of mutants was ruled out because the observed changes grossly occurred during a single subculture, after several subcultures in presence of the cell extract. Gross populational changes cannot be used as an accurate test of selection. The choice of genetic markers is important for interpretation of experimental data. In Streptomyces, antibiotic resistance may be coupled with antibiotic synthesis. Crossreaction among streptomyces-phages and the relatively high mutation rate from phage sensitivity to resistance makes this genetic marker difficult to score. Morphological expressions seemed to be related to antibiotic and bacteriophage sensitivity. Growth-factor requirements have not been found, in this study, to be coupled with morphology or susceptibility. Growth-factor requirements are invaluable in genetic studies of the Streptomyces.

This investigation was supported by a research grant from the National Institutes of Health, U.S. Public Health Service.

\section{REFERENCES}

Beadle, G. W. \& Coonradt, V. L. (1944). Heterokaryosis in Neurospora crassa. Genetics, 29, 291.

Bradley, S. G. (1957a). Heterokaryosis in Streptomyces coelicolor. J. Bact. 73, 581.

Bradley, S. G. (1957 b). Distribution of lysogenic Streptomyces. Science, 126, 558.

Bradley, S. G. $(1957 c)$. Induction of a lysogenic Streptomyces by a virulent bacteriophage. Bact. Proc. p. 40.

Bradiey, S. G. \& Lederberg, J. (1956). Heterokaryosis in Streptomyces. J. Bact. 72, 219. 
Braun, W., Firshein, W. \& Whallon, J. (1957). Effects of desoxyribonucleic acid breakdown products on bacterial population changes and virulence. Science, $125,445$.

Horvath, J., Marton, M. \& Oroszlan, I. (1954). Vegetative Hybridisationsversuche an Streptomyces. Acta Microbiol. Acad. Sci. Hungaricae, 2, 21.

Lederberg, J. (1952). Cell genetics and hereditary symbiosis. Physiol. Rev. 32, 403.

Mika, L., Braun, W., Ciaccio, E. \& Goodlow, R. (1954). The nature of the effect of $\alpha$-alanine on population changes of Brucella. J. Bact. 68,562 .

Sermonti, G. \& Spada-Sermonti, I. (1956). Gene recombination in Streptomyces coelicolor. J. gen. Microbiol. 15, 609.

Welsch, M. (1956). Evidence for the occurrence of true lysogeny among Actinomycetes. Virology, 2, 703.

(Received 28 October 1957) 